\title{
A STRONG FORM OF ALMOST DIFFERENTIABILITY
}

\author{
RICARDO ALMEIDA AND VÍTOR NEVES
}

\begin{abstract}
We present an uniformization of Reeken's macroscopic differentiability ([5]), discuss its relations to uniform differentiability ([6]) and classical continuous differentiability, prove corresponding Chain Rule, Taylor's Theorem, Mean Value Theorem and Inverse Mapping Theorem. An attempt at comparison with observability $([1,4])$ is made too.
\end{abstract}

\section{A SUMMARY}

In section 2 we establish the main context and language and also review Stroyan's S-uniform differentiability ([6]) for it not only is important for the matter at hand but also because we see it as a touchstone, or at least a basic paradigm, for other notions either finite or infinite dimensional. In section 3, we establish the uniformization we call $\mathbf{m u}$-differentibility (definition 3.3), and explore its relation to S-continuity (theorem 3.6), S-uniform differentiability (theorems 3.8 and 3.13) and classical continuous differentiability (theorem and 3.9 and corollary 3.11). Section 3 also includes a short discussion of mu-differentiability of higher order (theorem $3.12)$.

In sections 4, 5, 6 and 7 we treat the remaining theorems in the order given in the abstract.

In section 8 we sketch a line along which a comparison of observability and macroscopic differentiability might be studied.

\section{Preliminaries}

Our presentation is made in a poly-saturated model of Robinson's Nonstandard Analysis, as given for instance in [7] or [2]. Definitions and theorems in this introduction aim at making the article self-contained, at least on what regards terminology.

Unless otherwise specified, $E$ and $F$ are two arbitrary normed spaces with nonstandard extensions ${ }^{*} E$ and ${ }^{*} F$, and $U$ an open subset of $E$. We begin by presenting some basic notions and theorems.

Definition 2.1. Let $x$ and $y$ be two vectors of ${ }^{*} E$. We say that

(1) $x$ is infinitesimal if $|x|<r$ for all positive real numbers $r$, and we write $x \approx 0$;

(2) $x$ is finite if, for some positive real number $r,|x|<r$; the set of the finite vectors of ${ }^{*} E$ will be denoted by fin $\left({ }^{*} E\right)$;

(3) $x$ is infinite if it is not finite, and write $x \approx \infty$;

(4) $x$ and $y$ are infinitely close if $x-y$ is infinitesimal, and we write $x \approx y$;

2000 Mathematics Subject Classification: 26E35, 57R35, 46T20. Keywords and phrases: Nonstandard Analysis, m-differentiability.

The work was supported by Centre for Research on Optimization and Control (CEOC) from the "Fundação para a Ciência e a Tecnologia" FCT, cofinanced by the European Community Fund FEDER/POCTI. 
(5) $x$ is nearstandard if there exists a standard $z \in{ }^{\sigma} E$ with $x \approx z$, and we write $z=s t(x)$; in this case we say that $z$ is the standard part of $x$. The set of the nearstandard vectors of ${ }^{*} E$ will be denoted by $n s\left({ }^{*} E\right)$;

(6) The monad of $x$ is the set $\mu(x):=\left\{z \in{ }^{*} E \mid z \approx x\right\}$.

The set of infinitesimal vectors is the monad of zero. ${ }^{*} \mathbb{N}_{\infty}$ denotes the set of infinitely large positive integers, ${ }^{*} \mathbb{N}_{\infty}={ }^{*} \mathbb{N} \backslash \sigma \mathbb{N}$. ${ }^{*} \mathbb{Z}_{\infty}^{+},{ }^{*} \mathbb{Z}_{\infty}^{-},{ }^{*} \mathbb{Z}_{\infty},{ }^{*} \mathbb{R}_{\infty}$, etc are defined analogously.

Theorem 2.2. The inclusion $n s\left({ }^{*} E\right) \subseteq$ fin $\left({ }^{*} E\right)$ holds. Moreover, $E$ is finite dimensional if and only if $n s\left({ }^{*} E\right)=\operatorname{fin}\left({ }^{*} E\right)$.

In infinite dimensional spaces, finite vectors need not be nearstandard. For example, let $E=l_{1}(\mathbb{R})$ and take $x=\left(x_{n}\right) \in{ }^{*} l_{1}(\mathbb{R}),\left(n \in{ }^{*} \mathbb{N}\right)$ where

$$
x_{n}= \begin{cases}0 & n \neq \omega \\ 1 & n=\omega\end{cases}
$$

and $\omega \in{ }^{*} \mathbb{N}_{\infty}$. Then $x$ is finite $(|x|=1)$ but its distance to any standard element is not infinitesimal.

Theorem 2.3. Spillover Principle Let $A$ be an internal subset of ${ }^{*} \mathbb{R}$. If $A$ contains all positive infinitesimal numbers, then $A$ contains a positive standard number.

Definition 2.4. Let $f:{ }^{*} U \rightarrow{ }^{*} F$ be an internal function. We say that $f$ is $S$ continuous at $a \in{ }^{*} U$ if $x \approx a$ implies $f(x) \approx f(a)$. If this is true for all $a \in{ }^{\sigma} U$, $f$ is called $S$-continuous. If it still holds for all $a \in{ }^{*} U$, then we say that $f$ is $S U$-continuous.

Theorem 2.5. A standard function $f$ is continuous (resp. uniformly continuous) if and only if it is S-continuous (resp. SU-continuous).

For instance, $f(x)=x^{2}, x \in \mathbb{R}$ is not uniformly continuous since if $\omega$ is an infinite hyper-real number, then

$$
f\left(\omega+\frac{1}{\omega}\right)=\omega^{2}+\frac{1}{\omega^{2}}+2 \not \omega^{2}=f(\omega) .
$$

In the following we will denote

$$
n s\left({ }^{*} U\right):=\left\{x \in{ }^{*} U \mid x \in n s\left({ }^{*} E\right) \wedge s t(x) \in{ }^{\sigma} U\right\}
$$

Given an internal linear operator $L \in{ }^{*} L(E, F)$, we say that $L$ is finite if $L\left(f i n\left({ }^{*} E\right)\right) \subseteq \operatorname{fin}\left({ }^{*} F\right)$

Definition 2.6. Let $f:{ }^{*} U \rightarrow{ }^{*} F$ be an internal function and $a \in{ }^{\sigma} U$. We say that $f$ is $S$-differentiable at $a$ if it satisfies both conditions

(1) $f\left(n s\left({ }^{*} U\right)\right) \subseteq n s\left({ }^{*} F\right)$.

(2) there exists a finite linear operator $D f_{a} \in{ }^{*} L(E, F)$ such that, for each $x \approx a$ there exists some $\eta \approx 0$ satisfying

$$
f(x)-f(a)=D f_{a}(x-a)+|x-a| \eta
$$

We say that $f$ is a $S$-differentiable function if it is $S$-differentiable at all $a \in{ }^{\sigma} U$. Finally, we say that $f$ is $S U$-differentiable if the previous condition is true for every $a \in n s\left({ }^{*} U\right)$.

Theorem 2.7. A standard function $f: U \rightarrow F$ is differentiable (resp. continuously differentiable) if and only if it is $S$-differentiable (resp. SU-differentiable). 
Theorem 2.8. An internal function $f:{ }^{*} U \rightarrow{ }^{*} F$ is $S U$-differentiable if and only if for all $a \in{ }^{\sigma} U$, there exists a finite linear operator $L_{a} \in{ }^{*} L(E, F)$ such that, whenever $y \approx x \approx a$, there exists an infinitesimal vector $\eta$ satisfying

$$
f(x)-f(y)=L_{a}(x-y)+|x-y| \eta .
$$

It is well known that a real function of one real variable $f$ is differentiable with derivative $f^{\prime}$, then it is of class $C^{1}$ if and only if

$$
f^{\prime}(x) \approx \frac{f(x+\eta)-f(x)}{\eta}
$$

whenever $\eta$ is infinitesimal and $x$ is near standard (see $[7,5.7 .6]$ ); this idea is already extended in the following theorem (2.9) and was even more extended in [6].

We proceed to present a nonstandard version of Taylor's theorem. Note that it provides a necessary and sufficient condition for a function to be of class $C^{k}$. Denote $S L^{h}(E, F)$ the symmetric $h$-linear operators from $E \times \ldots \times E=E^{h}$ into $F$.

Theorem 2.9. Let $f: U \rightarrow F$ be a function. Then $f$ is of class $C^{k}$ if and only if there exist unique maps $L_{(.)}^{h}: U \rightarrow S L^{h}(E, F), h \in\{1, \ldots, k\}$ such that, whenever $a \in n s\left({ }^{*} U\right)$ and $x \approx a$, there is an infinitesimal $\eta \in{ }^{*} F$ satisfying

$$
f(x)=\sum_{h=0}^{k} \frac{1}{h !} L_{a}^{h}(x-a)^{(h)}+|x-a|^{k} \eta .
$$

The unique maps $L^{h}$ are the $h$-th derivatives of $f$ also denoted $D^{h} f$.

\section{MU-DIFFERENTIABILITY OF AN INTERNAL FUNCTION}

In this section treat a new kind of differentiability, we call mu-differentiability. We will see that mu-differentiability contrary to SU-differentiability demands less smoothness on $f$, but still approaches class $C^{1}$, namely when we deal with perturbations of classical functions (see Theorem 3.9 below).

In 1992, M. Reeken defined a new macroscopic differentiability (m-differentiability for short). The notion was used essentially for the definition of quasi-manifolds and we know of no developments other than the hereby presented. For standard functions, the m-derivative is the Fréchet derivative, but m-differentiability of internal functions does appear to be more adapted to physics ([5]).

Definition 3.1. Let $f:{ }^{*} U \rightarrow{ }^{*} F$ be an internal function. We say that $f$ is m-differentiable at $a \in{ }^{\sigma} U$ if it satisfies both conditions

(1) $f\left(n s\left({ }^{*} U\right)\right) \subseteq n s\left({ }^{*} F\right)$.

(2) there exists an infinitesimal $\delta_{a} \in{ }^{*} \mathbb{R}^{+}$and a finite linear operator $D f_{a} \in$ ${ }^{*} L(E, F)$ such that, for all $x \in{ }^{*} U$, where $\delta_{a}<|x-a| \approx 0$, there is some $\eta \approx 0$ such that

$$
f(x)-f(a)=D f_{a}(x-a)+|x-a| \eta
$$

The function $f$ is called $m$-differentiable if it is $m$-differentiable at all $a \in{ }^{\sigma} U$.

Since $f\left(n s\left({ }^{*} U\right)\right) \subseteq n s\left({ }^{*} F\right)$, it makes sense to define the standard function

$$
\begin{aligned}
s t(f):{ }^{\sigma} U & \rightarrow{ }^{\sigma} F \\
x & \mapsto s t(f(x))
\end{aligned}
$$

Let us denote $s t(f)$ by $\bar{f}$; this is merely a device to emphasize the fact that standard parts are actually extensions of classical objects, in particular the notation $s t(f)$ is bound to hide the fact that $s t(f)={ }^{*} g$, for some classical $g$. 
If $g$ is a standard differentiable function and $\sup _{x \in * U}|f(x)-g(x)| \approx 0$, then $f$ is m-differentiable. Actually, it can be proved that

Theorem 3.2. [5] If $E$ and $F$ are standard finite dimensional normed spaces, $K$ a standard compact subset of $E$ and $f:{ }^{*} K \rightarrow{ }^{*} F$ an internal function, then the following statements are equivalent:

(1) $f$ is $S$-continuous and $m$-differentiable;

(2) There exists a differentiable standard function $g: K \rightarrow F$ such that

$$
\sup _{x \in \in^{*} K}|f(x)-g(x)| \approx 0 .
$$

This result played a very important role in the characterization of nonstandard manifolds as presented in [5]: under some conditions, the internal transition functions $\varphi_{i j}$ are S-continuous, m-differentiable with S-continuous m-derivative if and only if there exist standard $C^{1}$ transition functions infinitely close to $\varphi_{i j}$.

Here we extend the last result for m-uniformly differentiable functions as well as study other properties of this differentiability. First we introduce the notion of mu-differentiability (short for m-uniformly differentiability).

Definition 3.3. Let $f:{ }^{*} U \rightarrow{ }^{*} F$ be an internal function. We say that $f$ is mu-differentiable if

(1) $f\left(n s\left({ }^{*} U\right)\right) \subseteq n s\left({ }^{*} F\right)$.

(2) There exists an internal function from ${ }^{*} U$ into ${ }^{*} L(E, F), x \mapsto D f_{x}$ such that

(a) when $x$ is near-standard in ${ }^{*} U, D f_{x}$ is a finite map.

(b) for each $a \in{ }^{\sigma} U$, there exists a positive infinitesimal $\delta_{a}$ for which, when $x, y \approx a \in{ }^{\sigma} U$, some infinitesimal vector $\eta$ verifies

$$
|x-y|>\delta_{a} \Rightarrow f(x)-f(y)=D f_{x}(x-y)+|x-y| \eta .
$$

Since $a \in \mu(a)$, every mu-differentiable function is m-differentiable.

For example, let

$$
f(x)= \begin{cases}0 & \text { if } x \neq 0 \\ \epsilon & \text { if } x=0\end{cases}
$$

where $\epsilon$ is a positive infinitesimal number. Then $f$ is mu-differentiable and (we can choose) $f^{\prime}(x) \approx 0$ for every $x \in n s\left({ }^{*} \mathbb{R}\right)$. In fact, let $a=0$ (the case $0 \neq a \in{ }^{\sigma} \mathbb{R}$ is obvious) and let $x \approx y \approx 0$ with $|x-y|>\delta_{0}:=\sqrt{\epsilon}$. Then

$$
\left|\frac{f(x)-f(y)}{x-y}\right| \leq \frac{\epsilon}{\sqrt{\epsilon}} \approx 0 .
$$

Observe that $f$ is not S-differentiable (nor SU-differentiable) since

$$
\frac{f\left(\epsilon^{2}\right)-f(0)}{\epsilon^{2}-0}=-\frac{\epsilon}{\epsilon^{2}}
$$

is infinite.

In the next example the choice of $\delta_{a}$ is independent of the choice of $a$.

Let $[x]$ denote the largest integer less than or equal to $x$. The function $f(x)=$ $[x] \epsilon, x \in{ }^{*} \mathbb{R}$, where $\epsilon$ is any positive infinitesimal, is mu-differentiable and $f^{\prime}(x)=0$, for every $x \in n s\left({ }^{*} \mathbb{R}\right)$. In fact, we suppose $a \in{ }^{\sigma} \mathbb{R}$ and choose a positive infinitesimal $\delta$ such that $\epsilon / \delta$ is still infinitesimal (for example, $\delta=\sqrt{\epsilon}$ ). If $x, y \approx a$ with $|x-y|>\delta$ then

(1) if $a \notin \mathbb{Z}$ then $\frac{f(x)-f(y)}{x-y}=0$;

(2) if $a \in \mathbb{Z}$ and $x, y \geq a$ or $x, y<a$ then $\frac{f(x)-f(y)}{x-y}=0$; 
(3) in the other cases, $\left|\frac{f(x)-f(y)}{x-y}\right| \leq \frac{\epsilon}{\delta} \approx 0$.

Actually one encompassing $\delta$ may be taken in Definition 3.3, i.e., the following holds.

Theorem 3.4. Let $f:{ }^{*} U \rightarrow{ }^{*} F$ be an internal function; $f$ is mu-differentiable if and only if all the following conditions are verified

(1) $f\left(n s\left({ }^{*} U\right)\right) \subseteq n s\left({ }^{*} F\right)$.

(2) There exist an internal function from ${ }^{*} U$ into ${ }^{*} L(E, F), x \mapsto D f_{x}$ and a positive infinitesimal $\delta$ such that

(a) when $x$ is near-standard in ${ }^{*} U, D f_{x}$ is a finite map.

(b) when $x$ and $y$ are near-standard in ${ }^{*} U$, some infinitesimal vector $\eta$ verifies

$$
|x-y|>\delta \Rightarrow f(x)-f(y)=D f_{x}(x-y)+|x-y| \eta .
$$

Proof. It is obvious that the existence of one $\delta$ as above implies mu-differentiability.

Suppose that $f$ is mu-differentiable as in Definition 3.3 and define

$$
\left.\left.\nu:=\bigcup_{a \in \in^{\sigma} U}\right] 0, \delta_{a}\right] .
$$

$\nu$ is a union of a family of internal sets whose cardinal does not exceed the cardinal of the contextual classical model of analysis from which the poly-saturated nonstandard model is obtained; let $\mu$ denote the monad of zero in ${ }^{*} \mathbb{R}$, so that $\nu \subseteq \mu$. The proof that actually $\nu \subset \mu$ is an easy exercise on poly-saturation. Any infinitesimal $\delta \in \mu \backslash \nu$ may be chosen.

The following is obvious

Theorem 3.5. Let $f$ and $g$ be two mu-differentiable functions and $k \in n s\left({ }^{*} \mathbb{R}\right)$. Then $f+g$ and $k f$ are mu-differentiable.

We shall prove

Theorem 3.6. If the function $f:{ }^{*} U \rightarrow{ }^{*} F$ is mu-differentiable then

$$
\forall x, y \in n s\left({ }^{*} U\right) \quad x \approx y \Rightarrow f(x) \approx f(y),
$$

i.e., the function is $S$-continuous.

Proof. Let us fix $x, y \in n s\left({ }^{*} U\right)$ with $x \approx y$ and let $a:=s t(x)$. Since $x, y \in \mu(a)$, there exist two finite linear operators $D f_{x}, D f_{y} \in{ }^{*} L(E, F)$ such that, for all $z \in$ $\mu(a)$

- $|x-z|>\delta_{a} \Rightarrow f(x)-f(z)=D f_{x}(x-z)+|x-z| \eta_{1}$,

- $|y-z|>\delta_{a} \Rightarrow f(y)-f(z)=D f_{y}(y-z)+|y-z| \eta_{2}$,

with $\eta_{1} \approx \eta_{2} \approx 0$. Choose any $z \in \mu(a)$ with $\min \{|x-z|,|y-z|\}>\delta_{a}$. Then

$$
f(x)-f(z) \approx 0 \approx f(y)-f(z)
$$

so that $f(x) \approx f(y)$, which concludes the proof.

Remark 3.7. m-differentiability of a function does not imply S-continuity. Let

$$
\begin{aligned}
& \left.f:{ }^{*}\right]-1,1\left[\longrightarrow{ }^{*} \mathbb{R}\right. \\
& x \mapsto\left\{\begin{array}{lll}
0 & \text { if } & x \neq \epsilon \\
1 & \text { if } & x=\epsilon
\end{array}\right.
\end{aligned}
$$

where $\epsilon$ is a positive infinitesimal number. Then $f$ is m-differentiable at $x=0$ (take $\left.\delta_{0} \geq \epsilon\right)$ but it is not S-continuous.

The next theorem shows that derivatives of mu-differentiable functions are Scontinuous: 
Theorem 3.8. Let $f$ be a mu-differentiable function, $x, y \in n s\left({ }^{*} U\right)$ with $x \approx y$. Then for all $d \in{ }^{*} E$ with $|d|=1, D f_{x}(d) \approx D f_{y}(d)$.

Proof. Let $a=s t(x)$ and $d \in{ }^{*} E$ with $|d|=1$. We will divide the proof in two cases. The first part of our proof is inspired by Stroyan's argument in the proof of [6, Proposition (2.4)].

First Case: $|x-y|>\delta_{a}$

Let $\epsilon:=\sqrt{|x-y|}$ and $z:=\epsilon d+x=\epsilon\left(d+\frac{x-y}{\epsilon}\right)+y$. Since

(1) $0 \approx|x-y|>\delta_{a}$;

(2) $0 \approx|z-x|=\epsilon>\delta_{a}$;

(3) $0 \approx|z-y| \geq \epsilon(1-\epsilon)>\delta_{a}$;

the following hold for some $\eta_{i}$ :

(1) $f(x)-f(y)=D f_{y}(x-y)+\epsilon \eta_{1}, \eta_{1} \approx 0$;

(2) $f(z)-f(x)=\epsilon D f_{x}(d)+\epsilon \eta_{2}, \eta_{2} \approx 0$

(3) $f(z)-f(y)=\epsilon D f_{y}(d)+D f_{y}(x-y)+\epsilon \eta_{3}, \eta_{3} \approx 0$.

So we conclude that, for some infinitesimal $\eta$,

$$
D f_{y}(x-y)+\epsilon \eta_{1}=f(x)-f(y)=\epsilon\left(D f_{y}(d)-D f_{x}(d)\right)+D f_{y}(x-y)+\epsilon \eta
$$

and thus $D f_{x}(d) \approx D f_{y}(d)$.

Second Case: $|x-y| \leq \delta_{a}$

Let $w \in{ }^{*} U$ be such that

$$
0 \approx|x-w|>\delta_{a} \quad \& \quad 0 \approx|y-w|>\delta_{a}
$$

Similarly to the first case, one can prove that for all $d \in{ }^{*} E$ with $|d|=1$

$$
D f_{x}(d) \approx D f_{w}(d) \approx D f_{y}(d) .
$$

We now present the main result of this chapter. It extends Theorem 3.2 for mu-differentiable functions. As one might expect, in this case, the internal function is infinitely close to a $C^{1}$ standard function.

Theorem 3.9. Let $f:{ }^{*} U \rightarrow{ }^{*} F$ be an internal function. Then:

(1) If $F$ is a finite dimensional space and $f$ is a mu-differentiable function, then $\bar{f}: U \rightarrow F$ is a $C^{1}$ function and $D \bar{f}_{a}=\operatorname{st}\left(D f_{a}\right)$ for $a \in{ }^{\sigma} U$. Furthermore, if $E$ is also finite dimensional then

$$
\forall a \in{ }^{\sigma} U \exists \eta_{0} \approx 0 \forall x \approx a \quad|f(x)-\bar{f}(x)| \leq \eta_{0} .
$$

(2) If there exists a $C^{1}$ standard function $g: U \rightarrow F$ with

$$
\forall a \in{ }^{\sigma} U \exists \eta_{0} \approx 0 \forall x \approx a \quad|f(x)-g(x)| \leq \eta_{0},
$$

then $f$ is mu-differentiable. Moreover, $g=\bar{f}$.

Proof. (1) Suppose that $F$ is a finite dimensional normed space and $f$ is mudifferentiable. We will begin by proving that $\bar{f}$ is classically differentiable at $a \in{ }^{\sigma} U$ with derivative $x \mapsto s t\left(D f_{a}(x)\right)$.

$$
\forall \eta \in{ }^{\sigma} \mathbb{R}^{+} \exists \epsilon \in{ }^{\sigma} \mathbb{R}^{+} \forall h \in{ }^{\sigma} E \quad 0<|h|<\epsilon \Rightarrow \frac{\left|\bar{f}(a+h)-\bar{f}(a)-s t\left(D f_{a}(h)\right)\right|}{|h|}<\eta .
$$

Fix $\eta \in{ }^{\sigma} \mathbb{R}^{+}$and let

$$
\begin{gathered}
A:=\left\{\epsilon \in{ }^{*} \mathbb{R}^{+} \mid \epsilon \leq \delta_{a} \vee\left[\forall h \in{ }^{*} E\right.\right. \\
\left.\left.\delta_{a}<|h|<\epsilon \Rightarrow \frac{\left|f(a+h)-f(a)-D f_{a}(h)\right|}{|h|}<\frac{\eta}{2}\right]\right\} .
\end{gathered}
$$


Since $A$ is an internal set and contains all positive infinitesimal numbers, by the Spillover Principle there exists $\epsilon \in{ }^{\sigma} \mathbb{R}^{+}$such that $\epsilon \in A$. Choose now $h \in{ }^{\sigma} E$ with $0<|h|<\epsilon$. As $h$ is standard, $\delta_{a}<|h|<\epsilon$; therefore

$$
\frac{\left|f(a+h)-f(a)-D f_{a}(h)\right|}{|h|}<\frac{\eta}{2} .
$$

Taking standard parts one gets

$$
\frac{\left|\bar{f}(a+h)-\bar{f}(a)-s t\left(D f_{a}(h)\right)\right|}{|h|}<\eta .
$$

So $\bar{f}$ is differentiable and $D \bar{f}_{a}=\operatorname{st}\left(D f_{a}\right)$ for $a \in{ }^{\sigma} U$.

Next we will prove that the function $x \mapsto D \bar{f}_{x}$ is classically continuous, i.e.,

$$
\begin{gathered}
\forall a \in{ }^{\sigma} U \forall \eta \in{ }^{\sigma} \mathbb{R}^{+} \exists \epsilon \in{ }^{\sigma} \mathbb{R}^{+} \forall x \in{ }^{\sigma} U \forall d \in{ }^{\sigma} E \\
{[|x-a|<\epsilon \wedge|d|=1] \Rightarrow\left|D \bar{f}_{x}(d)-D \bar{f}_{a}(d)\right|<\eta .}
\end{gathered}
$$

Choose any $a \in{ }^{\sigma} U$ and $\eta \in{ }^{\sigma} \mathbb{R}^{+}$and let

$$
\begin{gathered}
B:=\left\{\epsilon \in{ }^{*} \mathbb{R}^{+} \mid \forall x \in{ }^{*} U \forall d \in{ }^{*} E\right. \\
\left.[|x-a|<\epsilon \wedge|d|=1] \Rightarrow\left|D f_{x}(d)-D f_{a}(d)\right|<\frac{\eta}{2}\right\} .
\end{gathered}
$$

Again the internal set $B$ contains all positive infinitesimals. In fact, if $0<\epsilon \approx 0$, for any $x \in{ }^{*} U$ and $d \in{ }^{*} E$ with $|d|=1$ and $|x-a|<\epsilon$, by Theorem 3.8, one has $D f_{x}(d) \approx D f_{a}(d)$ and so

$$
\left|D f_{x}(d)-D f_{a}(d)\right|<\frac{\eta}{2}
$$

So $B$ must contain a positive standard $\epsilon$. Choose now $x \in{ }^{\sigma} U$ and $d \in{ }^{\sigma} E$ satisfying $|d|=1$ and $|x-a|<\epsilon$; hence

$$
\left|D f_{x}(d)-D f_{a}(d)\right|<\frac{\eta}{2}
$$

which implies

$$
\left|D \bar{f}_{x}(d)-D \bar{f}_{a}(d)\right|<\eta,
$$

proving that $\bar{f}$ is a $C^{1}$ function.

Assume now that $E$ is finite dimensional. Observe that for $a \in{ }^{\sigma} U$ and $x \approx a$, both $f(x) \approx f(a)$, by theorem 3.6, and $\bar{f}(x) \approx \bar{f}(a)$, as we just saw, therefore

$$
f(x)-\bar{f}(x) \approx f(a)-\bar{f}(a)=f(a)-s t(f(a)) \approx 0 .
$$

Therefore $f(x) \approx \bar{f}(x)$ for every $x \in n s\left({ }^{*} U\right)$.

Moreover, for every $a \in{ }^{\sigma} U$, we can choose $n \in{ }^{\sigma} \mathbb{N}$ such that $B_{2 / n}(a) \subseteq$ $U$. So, if we define $K$ as being the closed ball $\bar{B}_{1 / n}(a)$, we have

$$
a \in K \subseteq U .
$$

Let $y \in{ }^{*} K$. Since $K$ is compact, st $(y)$ belong to ${ }^{\sigma} K \subseteq{ }^{\sigma} U$. Define

$$
\eta_{0}:=\sup _{y \in * K}|f(y)-\bar{f}(y)| .
$$

It is easy to verify that $\eta_{0} \approx 0$, which ends the proof of 1 .

(2) Let $g \in C^{1}(U, F)$. Fix any $a \in{ }^{\sigma} U$ and let $\delta_{a}:=\sqrt{\eta_{0}}$. Choose any $x, y \in \mu(a)$ with $\delta_{a}<|x-y|$. Since $g$ is continuously differentiable, there exists a finite linear operator $D g_{x}$ which satisfies the condition

$$
g(x)-g(y)=D g_{x}(x-y)+|x-y| \eta
$$

for some $\eta \approx 0$. 
For $\epsilon_{1}:=g(x)-f(x)$ and $\epsilon_{2}:=g(y)-f(y)$, it is true that $\max \left\{\left|\epsilon_{1}\right|,\left|\epsilon_{2}\right|\right\} \leq$ $\eta_{0}$ and

$$
f(x)-f(y)=D g_{x}(x-y)+|x-y| \eta+\epsilon_{2}-\epsilon_{1} .
$$

Furthermore, we also have

$$
\frac{\left|\epsilon_{1}-\epsilon_{2}\right|}{|x-y|} \leq \frac{\left|\epsilon_{1}\right|+\left|\epsilon_{2}\right|}{|x-y|} \leq \frac{2 \eta_{0}}{\sqrt{\eta_{0}}} \approx 0 .
$$

To see that $g=\bar{f}$, note that both are standard functions and for every $a \in{ }^{\sigma} U, g(a)=\bar{f}(a)$.

Remark 3.10. The previous theorem is false if we replace mu-differentiability by SU-differentiability. Of course 1 still holds since SU-differentiability is a stronger condition, but 2 may fail. For example, suppose $g(x)=0, x \in \mathbb{R}$ and $f(x)=0$, if $x \in{ }^{*} \mathbb{R} \backslash\{0\}$ and $f(0)=\epsilon$ with $0 \neq \epsilon \in \mu(0)$. Then $g$ is a standard $C^{1}$ function infinitely close to $f$ but $f$ is not SU-differentiable.

It is easy to prove that

Corollary 3.11. For a standard function $f: U \rightarrow F$, the following conditions are equivalent:

(1) $f$ is of class $C^{1}$;

(2) $f$ is mu-differentiable.

A mu-differentiable function $f:{ }^{*} U \rightarrow{ }^{*} F$, internal, by definition, has an internal derivative $x \mapsto D f_{x} \in^{*} L(E, F)$, determined up to an infinitesimal map ([6]) called the mu-derivative of $f$. As $L(E, F)$ is still a standard normed space, we may define higher-order derivatives. We say that $f$ is twice mu-differentiable provided $f$ and $D f_{(\cdot)}$ are both mu-differentiable.

Recursively, $f$ is $k$-times mu-differentiable if $f$ is mu-differentiable and there exist mu-differentiable functions $D f_{(\cdot)}, \ldots, D^{k-1} f_{(\cdot)}$ such that all $D^{j} f_{(\cdot)}$ are a muderivative of $D^{j-1} f_{(\cdot)}, j=1, \ldots, k-1$.

Theorem 3.12. Let $f:{ }^{*} U \rightarrow{ }^{*} F$ be an internal function. Then:

(1) If $F$ is a finite dimensional space and $f$ is $k$-times mu-differentiable, then $\bar{f}: U \rightarrow F$ is a $C^{k}$ function and for each $a \in{ }^{\sigma} U, D^{j} \bar{f}_{a}=\operatorname{st}\left(D^{j} f_{a}\right)$ for $j=1,2, \ldots, k$. Furthermore, if $E$ is also finite dimensional,

$$
\forall a \in{ }^{\sigma} U \exists \eta_{0} \approx 0 \forall x \approx a \quad|f(x)-\bar{f}(x)| \leq \eta_{0}
$$

and

$$
\forall j \in\{1,2, \ldots, k-1\} \forall a \in{ }^{\sigma} U \exists \eta_{j} \approx 0 \forall x \approx a \quad\left|D^{j} f_{x}-D^{j} \bar{f}_{x}\right| \leq \eta_{j} .
$$

(2) If there exists a $C^{k}$ standard function $g: U \rightarrow F$ with

$$
\forall a \in{ }^{\sigma} U \exists \eta_{0} \approx 0 \forall x \approx a \quad|f(x)-g(x)| \leq \eta_{0}
$$

and

$\forall j \in\{1,2, \ldots, k-1\} \forall a \in{ }^{\sigma} U \exists \eta_{j} \approx 0 \forall x \approx a \quad\left|D^{j} f_{x}-D^{j} g_{x}\right| \leq \eta_{j}$

then $f$ is $k$-times mu-differentiable. Moreover, $g=\bar{f}$.

Proof. The proof is by induction on $k$ as follows:

For $k=1$ : it was proved in Theorem 3.9 that 1 and 2 hold.

For $k \Rightarrow k+1$ : 
We will begin by proving that 1 holds. Assume then that $f$ is $(k+1)$-times mu-differentiable. By hypothesis of induction, $\bar{f}$ is of class $C^{k}$ and satisfies the other conditions of 1 . Since

$$
\begin{aligned}
D^{k} f_{(\cdot)}:{ }^{*} U & \rightarrow{ }^{*} L^{k}(E, F) \\
x & \mapsto D^{k} f_{x}
\end{aligned}
$$

is still mu-differentiable, its standard part

$$
\begin{aligned}
\operatorname{st}\left(D^{k} f_{(\cdot)}\right):{ }^{\sigma} U & \rightarrow{ }^{\sigma} L^{k}(E, F) \\
x & \mapsto s t\left(D^{k} f_{x}\right)
\end{aligned}
$$

is of class $C^{1}$ and, for every $a \in{ }^{\sigma} U, \operatorname{Dst}\left(D^{k} f_{a}\right)=\operatorname{st}\left(D\left(D^{k} f_{a}\right)\right)$. But since, when $a$ is standard, $\operatorname{st}\left(D^{k} f_{a}\right)=D^{k} \bar{f}_{a}$,

- $D^{k} \bar{f}_{(\cdot)}$ is also of class $C^{1}$ and so $\bar{f}$ is of class $C^{k+1}$;

- $D^{k+1} \bar{f}_{a}=s t\left(D^{k+1} f_{a}\right)$.

Furthermore, for $a \in{ }^{\sigma} U$ and $x \approx a$,

$$
D^{k} f_{x} \approx D^{k} f_{a} \approx D^{k} \bar{f}_{a} \approx D^{k} \bar{f}_{x} .
$$

Similarly, as in the proof of Theorem 3.9 , we can prove that there exists an infinitesimal number $\eta_{k}$ for which holds

$$
\left|D^{k} f_{x}-D^{k} \bar{f}_{x}\right| \leq \eta_{k}
$$

whenever $x \approx a$ and $E$ is a finite dimensional normed space, which ends the first part of the proof.

To prove 2, assume that $g$ is a $C^{k+1}$ satisfying the conditions in 2. Then $f$ is $k$-times mu-differentiable. Besides this, $D^{k} g_{(\cdot)}$ is a $C^{1}$ function and

$$
\forall a \in{ }^{\sigma} U \exists \eta_{k} \approx 0 \forall x \approx a \quad\left|D^{k} f_{x}-D^{k} g_{x}\right| \leq \eta_{k} .
$$

By Theorem 3.9, $D^{k} f_{(\cdot)}$ is mu-differentiable and so $f$ is $(k+1)$-times mu-differentiable.

From the previous result one can see that there exist functions $k$-times mudifferentiable which are not $k$-times SU-differentiable. For example, let $f$ be the function defined in (1), pag. 4. Since $f$ is infinitely close to $g$, where $g(x):=$ $0, x \in \mathbb{R}$, and $g$ is of class $C^{k}$, then $f$ is $k$-times mu-differentiable yet is not SUdifferentiable.

The next theorem establishes a relation between mu-differentiability and a condition similar to SU-differentiability (see Definition 2.6).

Theorem 3.13. For every mu-differentiable function $f:{ }^{*} U \rightarrow{ }^{*} F$ we have

$$
\forall x \in n s\left({ }^{*} U\right) \exists \delta_{x} \approx 0 \exists D f_{x} \in{ }^{*} L(E, F) \forall y \in{ }^{*} U \exists \eta \approx 0
$$

$\left|D f_{x}\right|$ is finite $\wedge\left[\delta_{x}<|x-y| \approx 0 \Rightarrow f(x)-f(y)=D f_{x}(x-y)+|x-y| \eta\right]$.

Proof. For any $x \in n s\left({ }^{*} U\right)$, define $a:=s t(x)$ and $\delta_{x}:=\delta_{a}$. The proof follows easily.

The reverse of Theorem 3.13 is false, as shown in the following example.

Let $f$ be the real valued function

$$
f(x)= \begin{cases}x^{2} \sin \frac{1}{x} & \text { if } x \neq 0 \\ 0 & \text { if } x=0\end{cases}
$$

Since $f$ is not continuously differentiable, it can not be mu-differentiable. But it satisfies condition (2). Indeed, if $x \approx 0$ (the other cases are obvious), for $\delta_{x}:=|x|$ and $y \in{ }^{*} \mathbb{R}$ with $0 \approx|x-y|>\delta_{x}$, we get

$$
\frac{f(x)-f(y)}{x-y}=\frac{x^{2}}{x-y}\left(\sin \frac{1}{x}-\sin \frac{1}{y}\right)+\frac{x^{2}-y^{2}}{x-y} \sin \frac{1}{y} \approx 0
$$


since

$$
\left|\frac{x^{2}}{x-y}\right| \leq \frac{x^{2}}{|x|} \approx 0 \quad \& \quad \frac{x^{2}-y^{2}}{x-y}=x+y \approx 0 .
$$

As a consequence of the continuity of the derivative, we have (compare with Theorem 2.8)

Theorem 3.14. Let $f:{ }^{*} U \rightarrow{ }^{*} F$ be an internal function. Then conditions 1 and 2 are equivalent:

(1) $f$ is mu-differentiable.

(2) (a) $f\left(n s\left({ }^{*} U\right)\right) \subseteq n s\left({ }^{*} F\right)$.

(b)

$$
\forall a \in{ }^{\sigma} U \exists \delta_{a} \approx 0 \exists D f_{a} \in{ }^{*} L(E, F) \forall x, y \in \mu(a)
$$

$\left|D f_{a}\right|$ is finite $\wedge\left[|x-y|>\delta_{a} \Rightarrow f(x)-f(y)=D f_{a}(x-y)+|x-y| \eta\right]$ for some $\eta \approx 0$;

Proof. Let us fix $a \in{ }^{\sigma} U$ and $0<\delta_{a} \approx 0$ satisfying

$$
\forall x, y \in \mu(a) \quad|x-y|>\delta_{a} \Rightarrow \frac{f(x)-f(y)}{|x-y|} \approx D f_{x}\left(\frac{x-y}{|x-y|}\right) .
$$

By Theorem 3.8 it follows that

which proves that $1 \Rightarrow 2$.

$$
D f_{x}\left(\frac{x-y}{|x-y|}\right) \approx D f_{a}\left(\frac{x-y}{|x-y|}\right)
$$

To prove the converse, let $a \in{ }^{\sigma} U$ and $\delta_{a}$ as in $\mathcal{L}(a)$ Then, given $x \in \mu(a)$, define $D f_{x}:=D f_{a}$. The proof follows.

Theorem 3.15. If $f:{ }^{*} U \rightarrow{ }^{*} F$ is a mu-differentiable function, then for all standard $a \in{ }^{\sigma} U$, there exists a positive $\delta \approx 0$ such that, for all $d \in{ }^{*} E$ with $|d|=1$, there exists $k \in$ fin $\left({ }^{*} F\right)$ for which

$$
\forall x \in{ }^{*} U \quad x \approx a \Rightarrow \frac{f(x+\delta d)-f(x)}{\delta} \approx k
$$

holds.

Proof. Fix $a \in{ }^{\sigma} U$ and define $\delta:=2 \delta_{a}$. Fix an unit vector $d$ and let $k:=D f_{a}(d)$. Then for $x \approx a$

$$
\frac{f(x+\delta d)-f(x)}{\delta} \approx D f_{x}(d) \approx D f_{a}(d)=k .
$$

\section{The Chain Rule}

Mu-differentiable functions are m-differentiable, but not conversely thus making the latter a weaker notion, which nevertheless still verifies the chain rule below; actually the proof is generalizable to mu-differentiable functions (theorem 4.3).

Theorem 4.1. Chain Rule Let $g$ and $f$ be two $m$-differentiable functions at a and $g(a)$, respectively, where $a$ and $g(a)$ are two standard vectors. In addition, if $D g_{a}$ is invertible and $\left\|\left(D g_{a}\right)^{-1}\right\|$ is finite, then $f \circ g$ is $m$-differentiable at $a$ and $D(f \circ g)_{a}=D f_{g(a)} \circ D g_{a}$.

Proof. Define $\delta=\max \left\{\delta_{a}, 2 \delta_{g(a)}\left\|\left(D g_{a}\right)^{-1}\right\|\right\}$ and choose $x$ with $\delta<|x-a| \approx 0$.

Since $0 \approx|x-a|>\delta_{a}$ then $g(x) \approx g(a)$. On the other hand, for some $\eta_{1} \approx 0$,

$$
\begin{gathered}
|g(x)-g(a)|=\left|D g_{a}(x-a)+\right| x-a\left|\eta_{1}\right| \\
=|x-a|\left|D g_{a}\left(\frac{x-a}{|x-a|}\right)+\eta_{1}\right|>2 \delta_{g(a)}\left|\left(D g_{a}\right)^{-1}\right|\left|D g_{a}\left(\frac{x-a}{|x-a|}\right)+\eta_{1}\right| \geq
\end{gathered}
$$




$$
2 \delta_{g(a)}\left|\left(D g_{a}\right)^{-1}\left(D g_{a}\left(\frac{x-a}{|x-a|}\right)+\eta_{1}\right)\right|=2 \delta_{g(a)}\left|\frac{x-a}{|x-a|}+\left(D g_{a}\right)^{-1}\left(\eta_{1}\right)\right|>\delta_{g(a)} .
$$

So we conclude that $\delta_{g(a)}<|g(x)-g(a)| \approx 0$. Hence there exists $\eta_{2} \approx 0$ such that

$$
\begin{gathered}
f(g(x))-f(g(a))=D f_{g(a)}(g(x)-g(a))+|g(x)-g(a)| \eta_{2} \\
=D f_{g(a)}\left(D g_{a}(x-a)+|x-a| \eta_{1}\right)+\left|D g_{a}(x-a)+\right| x-a\left|\eta_{1}\right| \eta_{2} \\
=D f_{g(a)} D g_{a}(x-a)+|x-a|\left(D f_{g(a)}\left(\eta_{1}\right)+\left|D g_{a}\left(\frac{x-a}{|x-a|}\right)+\eta_{1}\right| \eta_{2}\right)
\end{gathered}
$$

with

$$
D f_{g(a)}\left(\eta_{1}\right)+\left|D g_{a}\left(\frac{x-a}{|x-a|}\right)+\eta_{1}\right| \eta_{2} \approx 0
$$

Remark 4.2. Suppose that $g$ and $f$ are two m-differentiable functions at $a$ and $g(a)$, respectively. This is not sufficient to guarantee that $f \circ g$ is also m-differentiable at $a$, as it will be shown in the following example.

Let $\epsilon$ be a positive infinitesimal,

$$
\begin{aligned}
g:{ }^{*} \mathbb{R} & \rightarrow{ }^{*} \mathbb{R} \\
x & \mapsto \epsilon x
\end{aligned}
$$

and

$$
\begin{aligned}
f: * \mathbb{R} & \rightarrow{ }^{*} \mathbb{R} \\
x & \mapsto\left\{\begin{array}{lll}
1 & \text { if } & 0<x<\epsilon \\
0 & \text { if } & x \leq 0 \vee x \geq \epsilon
\end{array}\right.
\end{aligned}
$$

It is easy to verify that $g$ is m-differentiable at $x=0$ and $f$ is m-differentiable at $g(0)=0$. But

$$
\begin{aligned}
f \circ g:{ }^{*} \mathbb{R} & \rightarrow \quad{ }^{*} \mathbb{R} \\
x & \mapsto\left\{\begin{array}{lll}
1 & \text { if } & 0<x<1 \\
0 & \text { if } & x \leq 0 \vee x \geq 1
\end{array}\right.
\end{aligned}
$$

is not m-differentiable at $x=0$.

Theorem 4.3. Chain Rule II Let $g$ and $f$ be two mu-differentiable functions. If $D g_{x}$ is invertible and $\left\|\left(D g_{x}\right)^{-1}\right\|$ is finite, whenever $x$ is nearstandard, then $f \circ g$ is mu-differentiable and $D(f \circ g)_{x}=D f_{g(x)} \circ D g_{x}$.

Proof. Sketch of proof: To make it simple, denote $\delta_{f}$ and $\delta_{g}$ the infinitesimals as in Theorem 3.4 (with obvious meanings). Given a nearstandard $x$, let $\delta:=$ $\max \left\{\delta_{g}, 2 \delta_{f}\left\|\left(D g_{x}\right)^{-1}\right\|\right\}$. Replacing $a$ by $x$ and $x$ by $y$ (where $y \approx x$ ) in the proof of Theorem 4.1, the proof follows.

\section{TAYLOR'S TheOREM}

We can now formulate Taylor's Theorem for a mu-differentiable function defined on finite dimensional spaces. We will prove two different versions of this theorem; the first Taylor's expansion is made with internal functions and the second with standard functions.

Theorem 5.1. Taylor's Theorem Let $E$ and $F$ be two standard finite dimensional spaces, $U \subset E$ a standard open set and $f:{ }^{*} U \rightarrow{ }^{*} F$ an internal function $k$-times mu-differentiable, for some $k \in{ }^{\sigma} \mathbb{N}$. Then, 
(1) for every $x \in n s\left({ }^{*} U\right)$, there exists $\epsilon \approx 0$ such that, whenever $y \in{ }^{*} U$ with $\epsilon<|y-x| \approx 0$, there exists $\eta \approx 0$ satisfying

$f(y)=f(x)+D f_{x}(y-x)+\frac{1}{2 !} D^{2} f_{x}(y-x)^{(2)}+\ldots+\frac{1}{k !} D^{k} f_{x}(y-x)^{(k)}+|y-x|^{k} \eta$.

(2) for every $x \in n s\left({ }^{*} U\right)$, there exists $\epsilon \approx 0$ such that, whenever $y \in{ }^{*} U$ with $\epsilon<|y-x| \approx 0$, there exists $\eta \approx 0$ satisfying

$$
\begin{gathered}
f(y)=\bar{f}(x)+D \bar{f}_{x}(y-x)+\frac{1}{2 !} D^{2} \bar{f}_{x}(y-x)^{(2)}+\ldots \\
+\frac{1}{k !} D^{k} \bar{f}_{x}(y-x)^{(k)}+|y-x|^{k} \eta .
\end{gathered}
$$

Proof. (1) Let us begin by fixing $x \in n s\left({ }^{*} U\right)$ and let $a:=s t(x) \in{ }^{\sigma} U$. By Theorem 3.12, we know that $\bar{f}$ is of class $C^{k}$,

$$
\exists \eta_{0} \approx 0 \forall y \approx a \quad|f(y)-\bar{f}(y)| \leq \eta_{0}
$$

and for each $j=1,2, \ldots, k-1$,

$\exists \eta_{j} \approx 0 \forall y \approx a \quad \sup _{d_{i} \in \in^{*},\left|d_{i}\right|=1}\left|D^{j} f_{y}\left(d_{1}, \ldots, d_{j}\right)-D^{j} \bar{f}_{y}\left(d_{1}, \ldots, d_{j}\right)\right| \leq \eta_{j}$.

Define $\epsilon=\max \left\{\eta_{0}^{\frac{1}{k+1}}, \eta_{1}^{\frac{1}{k}}, \ldots, \eta_{k-1}^{\frac{1}{2}}\right\}$ and take $y \in{ }^{*} U$ with $\epsilon<|y-x| \approx 0$.

Define the finite sequence $\left(\epsilon_{i}\right)_{i=-1, \ldots, k-1}$ by

- $f(y)=\bar{f}(y)+\epsilon_{-1}$,

- $f(x)=\bar{f}(x)+\epsilon_{0}$,

- $D f_{x}(y-x)=D \bar{f}_{x}(y-x)+|y-x| \epsilon_{1}$,

- $D^{2} f_{x}(y-x)^{(2)}=D^{2} \bar{f}_{x}(y-x)^{(2)}+|y-x|^{2} \epsilon_{2}$,

- ...

- $D^{k-1} f_{x}(y-x)^{(k-1)}=D^{k-1} \bar{f}_{x}(y-x)^{(k-1)}+|y-x|^{k-1} \epsilon_{k-1}$.

Furthermore, since the maps $x \mapsto D^{k} f_{x}$ and $x \mapsto D^{k} s t(f)_{x}$ are both S-continuous, we also have

$$
\begin{gathered}
D^{k} f_{x}\left(\frac{y-x}{|y-x|}\right)^{(k)} \approx D^{k} f_{a}\left(\frac{y-x}{|y-x|}\right)^{(k)} \approx \\
D^{k} \bar{f}_{a}\left(\frac{y-x}{|y-x|}\right)^{(k)} \approx D^{k} \bar{f}_{x}\left(\frac{y-x}{|y-x|}\right)^{(k)},
\end{gathered}
$$

so there exists $\epsilon_{k} \approx 0$ with

$$
D^{k} f_{x}(y-x)^{(k)}=D^{k} \bar{f}_{x}(y-x)^{(k)}+|y-x|^{k} \epsilon_{k} .
$$

Using the fact that $\bar{f}$ is a $C^{k}$ function, one has

$$
\begin{gathered}
\bar{f}(y)=\bar{f}(x)+D \bar{f}_{x}(y-x)+\frac{1}{2 !} D^{2} \bar{f}_{x}(y-x)^{(2)}+\ldots \\
+\frac{1}{k !} D^{k} \bar{f}_{x}(y-x)^{(k)}+|y-x|^{k} \eta
\end{gathered}
$$

that is

$$
\begin{aligned}
f(y)= & f(x)+D f_{x}(y-x)+\frac{1}{2 !} D^{2} f_{x}(y-x)^{(2)}+\ldots+\frac{1}{k !} D^{k} f_{x}(y-x)^{(k)}+|y-x|^{k} \eta \\
& +\epsilon_{-1}-\epsilon_{0}-|y-x| \epsilon_{1}-|y-x|^{2} \epsilon_{2}-\ldots-|y-x|^{k-1} \epsilon_{k-1}-|y-x|^{k} \epsilon_{k} . \\
& \quad \text { If } \\
& \epsilon_{-1}-\epsilon_{0}-|y-x| \epsilon_{1}-|y-x|^{2} \epsilon_{2}-\ldots-|y-x|^{k-1} \epsilon_{k-1}=|y-x|^{k} \eta_{1},
\end{aligned}
$$

then $\eta_{1}$ is infinitesimal since

$\left|\eta_{1}\right| \leq \frac{\left|\epsilon_{-1}\right|}{|y-x|^{k}}+\frac{\left|\epsilon_{0}\right|}{|y-x|^{k}}+\frac{\left|\epsilon_{1}\right|}{|y-x|^{k-1}}+\frac{\left|\epsilon_{2}\right|}{|y-x|^{k-2}}+\ldots+\frac{\left|\epsilon_{k-1}\right|}{|y-x|}$ 


$$
\leq \frac{\eta_{0}}{\eta_{0}^{\frac{k}{k+1}}}+\frac{\eta_{0}}{\eta_{0}^{\frac{k}{k+1}}}+\frac{\eta_{1}}{\eta_{1}^{\frac{k-1}{k}}}+\frac{\eta_{2}}{\eta_{2}^{\frac{k-2}{k-1}}}+\ldots+\frac{\eta_{k-1}}{\eta_{k-1}^{\frac{1}{2}}} \approx 0 .
$$

(2) Analogously, if we take $\epsilon:=\eta_{0}^{\frac{1}{k+1}}$, the result follows.

\section{The Mean Value Theorem}

We give now a Mean Value Theorem for mu-differentiable functions.

Theorem 6.1. Mean Value Theorem Let $U$ be a standard open convex subset of $E$ and $f:{ }^{*} U \rightarrow{ }^{*} \mathbb{R}$ an internal mu-differentiable function. Take $\delta$ as given by Theorem 3.4. Then, for all $x, y \in n s\left({ }^{*} U\right)$ with $|x-y|>\delta$,

$$
\exists c \in[x, y] \quad f(x)-f(y)=D f_{c}(x-y)+|x-y| \eta
$$

for some $\eta \approx 0$.

Proof. If $x \approx y$, it is clear. If not, define a hyper-finite sequence $\left\{x_{n} \mid n \in\right.$ $\{1, \ldots, N+1\}\}$ by the formula

$$
x_{n}:=x+(n-1) \frac{y-x}{N},
$$

where $N \in{ }^{*} \mathbb{N}_{\infty}$ and $N<\frac{|y-x|}{\delta} \approx \infty$.

Then

$$
\begin{gathered}
f(x)-f(y)=\sum_{n=1}^{N}\left(f\left(x_{n}\right)-f\left(x_{n+1}\right)\right)= \\
=\sum_{n=1}^{N} D f_{x_{n}}\left(x_{n}-x_{n+1}\right)+\sum_{n=1}^{N}\left|x_{n}-x_{n+1}\right| \eta_{n} .
\end{gathered}
$$

If

$$
\sum_{n=1}^{N}\left|x_{n}-x_{n+1}\right| \eta_{n}=|y-x| \eta
$$

for some $\eta$, then $\eta \approx 0$. Indeed, by the convexity property of the norm

$$
|\eta| \leq \frac{\sum_{n=1}^{N}\left|x_{n}-x_{n+1}\right|\left|\eta_{n}\right|}{|y-x|}=\frac{\sum_{n=1}^{N}\left|x_{n}-x_{n+1}\right|\left|\eta_{n}\right|}{\sum_{n=1}^{N}\left|x_{n}-x_{n+1}\right|} \leq \max _{n \in\{1, \ldots, N\}}\left\{\left|\eta_{n}\right|\right\} \approx 0 .
$$

We will prove now that there exists $c \in[x, y]$ such that

$$
D f_{c}\left(\frac{x-y}{|x-y|}\right) \approx \frac{\sum_{n=1}^{N} D f_{x_{n}}\left(x_{n}-x_{n+1}\right)}{|x-y|} .
$$

Letting $d:=\frac{x-y}{|x-y|}$, it is true that

$$
\frac{\sum_{n=1}^{N} D f_{x_{n}}\left(x_{n}-x_{n+1}\right)}{|x-y|}=\frac{\sum_{n=1}^{N} D f_{x_{n}}\left(x_{n}-x_{n+1}\right)}{\sum_{n=1}^{N}\left|x_{n}-x_{n+1}\right|}=\frac{\sum_{n=1}^{N} D f_{x_{n}}(d)}{N} .
$$

Choosing $m, M \in\left\{x_{1}, \ldots, x_{N}\right\}$ with

$$
D f_{m}(d)=\min _{1 \leq n \leq N} D f_{x_{n}}(d) \quad \& \quad D f_{M}(d)=\max _{1 \leq n \leq N} D f_{x_{n}}(d),
$$

we get

$$
D f_{m}(d) \leq \frac{\sum_{n=1}^{N} D f_{x_{n}}(d)}{N} \leq D f_{M}(d) .
$$

So, there exists $c \in[m, M] \subseteq[x, y]$ with

$$
D f_{c}(d) \approx \frac{\sum_{n=1}^{N} D f_{x_{n}}(d)}{N} .
$$


We can formulate Theorem 6.1 for functions taking values in a normed space:

Theorem 6.2. Let $U$ be a standard open convex subset of $E$ and $f:{ }^{*} U \rightarrow{ }^{*} F$ an internal mu-differentiable function. Take $\delta$ as given by Theorem 3.4. Then, for all $x, y \in n s\left({ }^{*} U\right)$ with $|x-y|>\delta$,

$$
\exists c \in[x, y] \quad|f(x)-f(y)| \leq\left|D f_{c}(x-y)\right|+|x-y| \eta
$$

for some $\eta \approx 0$.

Proof. Following the proof of Theorem 6.1, it is true that:

For some $\eta_{1}, \ldots, \eta_{N} \approx 0$,

$$
\begin{gathered}
|f(x)-f(y)|=\left|\sum_{n=1}^{N}\left(f\left(x_{n}\right)-f\left(x_{n+1}\right)\right)\right| \\
\leq \sum_{n=1}^{N}\left|f\left(x_{n}\right)-f\left(x_{n+1}\right)\right|=\sum_{n=1}^{N}\left|D f_{x_{n}}\left(x_{n}-x_{n+1}\right)+\right| x_{n}-x_{n+1}\left|\eta_{n}\right| \\
\leq \sum_{n=1}^{N}\left|D f_{x_{n}}\left(x_{n}-x_{n+1}\right)\right|+\sum_{n=1}^{N}\left|x_{n}-x_{n+1}\right| \cdot\left|\eta_{n}\right| .
\end{gathered}
$$

Again, choose $m, M \in\left\{x_{1}, \ldots, x_{N}\right\}$ with

$$
\left|D f_{m}(d)\right|=\min _{1 \leq n \leq N}\left|D f_{x_{n}}(d)\right| \quad \& \quad\left|D f_{M}(d)\right|=\max _{1 \leq n \leq N}\left|D f_{x_{n}}(d)\right| .
$$

Since

there exists $c \in[x, y]$ with

$$
\left|D f_{m}(d)\right| \leq \frac{\sum_{n=1}^{N}\left|D f_{x_{n}}(d)\right|}{N} \leq\left|D f_{M}(d)\right|
$$

$$
\left|D f_{c}(d)\right| \approx \frac{\sum_{n=1}^{N}\left|D f_{x_{n}}(d)\right|}{N}
$$

\section{The Inverse Mapping Theorem}

A full Inverse Mapping Theorem is not expected. In fact, take for example the $C^{1}$ function $g(x)=x$. By Theorem 3.9, any internal function infinitely close to $g$ is mu-differentiable. So the $1-1$ condition may easily fail. Nevertheless, we have some form of injectivity as the next theorem states.

Theorem 7.1. Inverse Mapping Theorem Let $f:{ }^{*} U \rightarrow{ }^{*} F$ be an internal mu-differentiable function. Assume that, for a certain $a \in{ }^{\sigma} U, D f_{a}$ is invertible and $\left\|\left(D f_{a}\right)^{-1}\right\|$ is finite. Then there exists a standard neighborhood ${ }^{*} V$ of a such that $f$ is 1-to-1 on the standard elements of ${ }^{*} V$, i.e.,

$$
\forall x, y \in{ }^{\sigma} V \quad x \neq y \Rightarrow f(x) \neq f(y) .
$$

Proof. Let

$$
A:=\left\{\epsilon \in{ }^{*} \mathbb{R}^{+}\left|\forall x, y \in B_{\epsilon}(a) \quad\right| x-y \mid>\delta_{a} \Rightarrow f(x) \neq f(y)\right\} .
$$

Then $A$ contains all positive infinitesimal numbers since, for $0<\epsilon \approx 0$ and $x, y \in$ $B_{\epsilon}(a)$ with $|x-y|>\delta_{a}$, by Theorem 3.14 ,

$$
\frac{f(x)-f(y)}{|x-y|} \approx D f_{a}\left(\frac{x-y}{|x-y|}\right) .
$$


But

$$
1=\left|\left(D f_{a}\right)^{-1} D f_{a}\left(\frac{x-y}{|x-y|}\right)\right| \leq\left\|\left(D f_{a}\right)^{-1}\right\|\left|D f_{a}\left(\frac{x-y}{|x-y|}\right)\right| .
$$

Consequently,

$$
\left|D f_{a}\left(\frac{x-y}{|x-y|}\right)\right| \geq \frac{1}{\left\|\left(D f_{a}\right)^{-1}\right\|} \not \approx 0 .
$$

Therefore $f(x) \neq f(y)$. Using the Spillover Principle we can guarantee the existence of $\epsilon \in{ }^{\sigma} \mathbb{R}$ with $\epsilon \in A$. Define $V:=B_{\epsilon}(a)$ and take two standard elements of ${ }^{*} V$ with $x \neq y$. Since the distance between two distinct standard vectors is always greater than any infinitesimal number, one obtains $f(x) \neq f(y)$.

Remark 7.2. With the previous conditions we can not conclude that $f$ is 1 -to- 1 on ${ }^{*} V$. In fact, consider

$$
f(x)= \begin{cases}x & \text { if } x \neq 0 \\ \epsilon & \text { if } x=0\end{cases}
$$

where $\epsilon$ is any non-zero infinitesimal number. This function is mu-differentiable (it is infinitely close to $g(x)=x$ ) but it is never injective in any standard neighborhood of zero.

\section{A Note on OBSERVABle FUnCtions}

Harthong defined, and together with Reder treated observable functions (see $[1,4])$. Although that concept was presented and treated in the context of Internal Set Theory, there might a bridge between observable and observation functions on the one hand and mu-differentiable functions on the other hand, in that a function might be "strongly observable" if and only if its anti-derivative is m-differentiable.

\section{ACKNOWLEDGEMENTS}

We thank the referee not only for his suggestions for improvement of Definition 3.3 as well as for corrections on the proofs of Theorems 3.4 and 6.1. We are also grateful for his having brought to our attention the notions of observability as well as for his more specific comments which motivated practically all scientific aspects of the sketchy discussion in section 8 .

\section{REFERENCES}

[1] J. Harthong, Le moire, Adv. in Appl. Math., 2 (1), (1981): 24-75.

[2] A.E. Hurd, P.A. LoeB, An introduction to nonstandard real analysis, Academic Press, 1985.

[3] V. Neves, Nonstandard Calculus of Variations - a survey, J. Math. Sci. (Series of Contemporary Mathematics and Its Applications, Special volume "Aveiro Seminar on Control, Optimization, and Graph Theory"), 120 (1), (2004): 940-954.

[4] C. Reder, Observation macroscopique de phénomènes microscopiques, Actes de l'École d'Été: Analyse Non Standard et Représentation du Réel (Oran, 1984): 195-244, OPU, Algiers, 1985.

[5] K. Schlesinger, Generalized manifolds, Addison Wesley Longman, 1997.

[6] K. Stroyan, Infinitesimal calculus on locally convex spaces: 1. Fundamentals, Trans. Amer. Math. Soc., 240, (1978): 363-383.

[7] K. Stroyan, W. Luxemburg, Introduction to the theory of infinitesimals, Academic Press, 1976.

[8] A. Troesch, E. Urlacher, Perturbations singulières et analyse non standard, C. R. Acad. Sci. Paris Sér. A-B, 287 (14), (1978).

[9] E. URlacher, Un système rapidement oscillant, Mathématiques finitaires et analyse non standard, 1 (2): 235-256, Publ. Math. Univ. Paris VII, 31, Univ. Paris VII, Paris, 1989. 
Departamento de Matemática,

Universidade de Aveiro

3810-193 Aveiro - Portugal

E-mail address: ricardo.almeida@ua.pt

Departamento de Matemática,

Universidade de Aveiro

3810-193 Aveiro - Portugal

E-mail address: vneves@ua.pt 